\title{
Modeling of sound-structure interaction in a rectangular enclosure using finite element method
}

\begin{abstract}
In this paper the effect of periodic noise traveling into a rectangular enclosure is simulated using finite element technique using COMSOL Multiphysics software. The acoustic wave which is generated by a point source outside an enclosure propagates through the enclosure wall and excites the flexible panel which is mounted on the enclosure. The behavior of transmission of sound into the cavity is investigated by computing the modal characteristics of the cavity. Base on the model proposed, simulation part is conducted to examine the natural frequency response of system as well as to compute the distortion effect of the structure response and the acoustic pressure observed within the enclosure. The simulation results were compared with previous experimental and analytical works for validation and a good match between them were obtained.
\end{abstract}

Keyword: Acoustic-structure coupling; COMSOL; Noise and vibration 